\title{
Forecasting of the Photovoltaic Electricity Production on a Sail Ship by Taking Account Shadow Effects
}

\author{
B. Genet ${ }^{1}$, V. Boitier ${ }^{1 *}, Y$. Briere ${ }^{2}$, and F. Defaÿ ${ }^{2}$ \\ ${ }^{1}$ CNRS, LAAS, 7 Avenue du colonel Roche, F-31400 Toulouse, France. \\ 2 ISAE-SUPAERO, 10 Avenue Edouard Belin, F-31400 Toulouse, France. \\ *Contact author : vincent.boitier@laas.fr, +33 561336231
}

\begin{abstract}
Autonomous sailing robots have virtually no duration limit in operation because the main source of energy, for propulsion, is the wind. This paper focuses on the IBOAT3, a 1.8 meters long sailing robot ship developed at ISAE-SUPAERO. The onboard electronic consumption (actuators, GPS, ...) is supplied by a Li-ion battery. A $100 \mathrm{~W}$ photovoltaic panel mounted on the roof is used to recharge the battery. Unfortunately, shadows of the sail decrease the amount of energy given by the sun. This paper deals with the forecasting of the harvestable electric power taking account the trajectory, the wind and different factors that may have an impact on the journey across the ocean.
\end{abstract}

Key words: Irradiance Model, Partial Shading, Sailing Robot, Energy Harvesting

\section{Introduction}

Ocean observation is based on a mitigation of data collected via various systems: satellites, fixed buoys, drifting buoys, research ships, underwater autonomous vehicles, etc. Understanding fluids and energy exchange at sea surface is a key issue for climatology and ocean science. Autonomous sailing robots, compared to fixed buoys, or passive drifting buoys, have the capability of precise localization and time sampling. Being propelled by wind have virtually no limit in autonomy. The few amount of power needed (typically a few watts to control rudder orientation and power computer and communication systems) can easily be provided by solar panels. The IBOAT project [1] investigates the design and control of Autonomous Sailing Robots, compatible with observation mission, but initially designed to run the Microtransat Challenge [2]. IBOAT 3 is the last prototype (Fig. 1). The required electrical power comes from a lithium-ion battery and a photovoltaic panel mounted on the top of it. Solar energy on boats is generally used for auxiliary equipment [3] or for propelling boats that have small crew [4], but no paper to our knowledge studies the impact of a sail shadow on the production of electricity. Indeed the waves and wind causes movements and oscillations on the boat. Those movements, coupled with the relative position of the sail and the sun, may create a partial shadow on the solar panel and therefore a drop of electrical production [5]. This paper traces the different steps needed to quantify that impact.

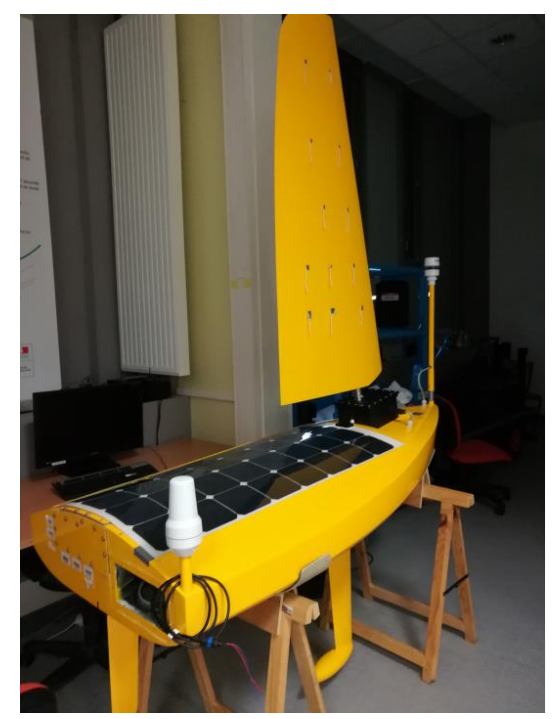

Fig. 1. IBOAT3 is a fully autonomous sailing boat. Length: 1.8 $\mathrm{m}$, height: $2 \mathrm{~m}$, weight, $35 \mathrm{~kg}$. Electrical power is provided by a $0.65 \mathrm{~m}^{2}$ solar panel

In a first part, we calculate the solar irradiance on a tilted surface using models and meteorological data. In the second part, we compute the shadow of the sail on the solar panel in order to deduce the percentage of shaded surface. Then in the third part, we calculate the total power produced by the whole panel. In part 5 , we present the behavior model of the boat to determine its speed, heading, and the sail angle. Finally, in part 6, we compare the total energy budget of our full model relatively to a generic model.

\section{Computing the solar irradiance}

The solar irradiance is the power per unit of surface received from the Sun. It is made of three components represented on Fig. 2:

- The direct component, $I_{\text {direct }}$, coming directly from the Sun

- The diffuse component, $I_{\text {diffuse }}$, coming from all the direction of the sky but the Sun

- The reflected component, $I_{\text {reflect }}$, coming, in the case of a boat, from the ocean 
Note that even if a ray has been deflected multiple times, it will be considered part of the direct component if its final direction is the same as the sun.

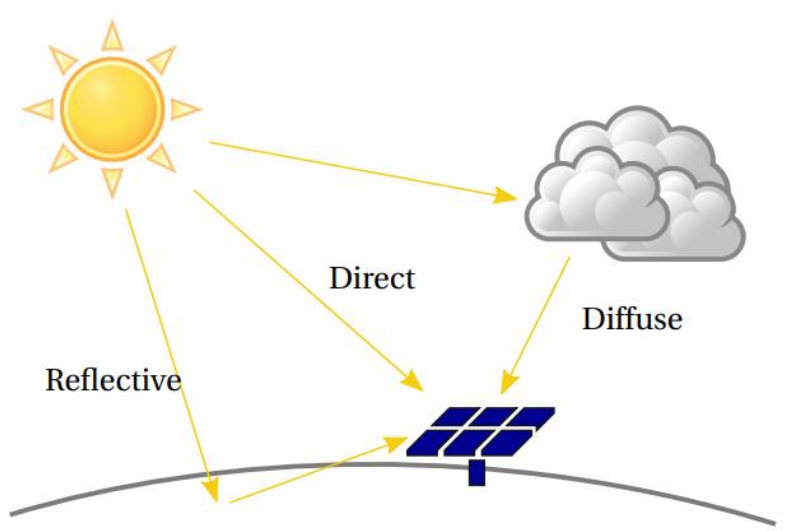

Fig. 2. The three components of the Sun light

To determine the solar irradiance on a given point we use the model [6]. This model needs two inputs: the solar irradiance on top of atmosphere $\left(\mathrm{H}_{0}\right)$, also called TOA irradiance, and the global irradiance on a horizontal surface on the ground $\left(\mathrm{I}_{0}\right)$. Those two values can be computed using irradiation values from a NASA database (power.larc.nasa.gov website). The model can determine the distribution of irradiance among its three components using the upcoming variables:

- $k_{t}=\frac{I_{0}}{H_{0}}$ the clarity of the sky ( 1 is a very clear sky)

- $d_{t}$ the percentage of diffuse light (and $1-d_{t}$ is the percentage of direct light)

- $\Theta$ the angle between the normal vector of the surface and the direction of the sun in radians

- $\quad h$ the elevation of the sun

The percentage of diffuse light is related to the clarity of the sky according to the empirical model $d_{t}=\frac{1}{1+e^{5+8.6 k_{t}}}$ [6].

\section{A. Direct component}

The direct component of the irradiation is shown on (1).

$$
I_{\text {direct }}=I_{0}\left(1-d_{t}\right) \cos \Theta \sin h
$$

\section{B. Diffuse component}

To compute the diffuse component we then combine the effect of the sail and the model developed by [7] who divides the diffuse light into three components: an isotropic one, one to describe the circumsolar directions and one for the area close to horizon (2).

$$
\begin{gathered}
I_{\text {diffuse }}= \\
I_{\text {diff } 0}(h)\left[\begin{array}{c}
\frac{1+\cos i}{2}\left(1-F_{1}\right)+F_{1} \frac{a_{1}}{a_{2}}+F_{2} \sin i \\
-\psi]
\end{array}\right.
\end{gathered}
$$

Where:

- $\quad I_{\text {diff } 0}(h)=I_{0} d_{t} \sin h$

- $\quad i$ is the tilted angle of the panel, a horizontal panel will have a tilt angle of $0^{\circ}$

- $\Psi$ is the percentage of the sky that is hidden by the sail

- $a_{1}=\max (0, \cos \Theta)$

- $a_{2}=\max \left(\cos 85^{\circ}, \cos \Theta\right)$

- $\quad F_{1}=\max \left(0,\left[F_{11}+F_{12} \Delta+F_{13} \Theta\right]\right)$

- $F_{2}=F_{21}+F_{22} \Delta+F_{23} \Theta$

- $\Delta=m \frac{I_{\text {diff } 0}(h)}{I_{0} \sin h}$

- $m=\frac{1}{\cos \theta}$

$F_{i j}$ are tabulated values in Table I depending on $\varepsilon(3)$.

$$
\varepsilon=\frac{\frac{I_{\text {diff } 0}(h)+I_{\text {direct } 0}(h)}{I_{\text {diff } 0}(h)}+k \Theta^{3}}{1+k \Theta^{3}}
$$

Where $k=5.53510^{-6} S I$.

Table I. - $F_{i j}$ values for different $\varepsilon$, source [7]

\begin{tabular}{l|cccccc}
$\varepsilon$ & $F_{11}$ & $F_{12}$ & $F_{13}$ & $F_{21}$ & $F_{22}$ & $F_{23}$ \\
\hline 1.000 & -0.008 & 0.588 & -0.062 & -0.060 & 0.072 & -0.022 \\
1.065 & 0.130 & 0.683 & -0.151 & -0.019 & 0.066 & -0.029 \\
1.230 & 0.330 & 0.487 & -0.221 & 0.055 & -0.064 & -0.026 \\
1.500 & 0.568 & 0.187 & -0.295 & 0.109 & -0.152 & 0.014 \\
1.950 & 0.873 & -0.392 & -0.362 & 0.226 & -0.462 & 0.001 \\
2.800 & 1.132 & -1.237 & -0.412 & 0.288 & -0.823 & 0.056 \\
4.500 & 1.060 & -1.600 & -0.359 & 0.264 & -1.127 & 0.131 \\
6.300 & 0.678 & -0.327 & -0.250 & 0.159 & -1.377 & 0.251 \\
\hline
\end{tabular}

\section{Reflective component}

The last component is due to ocean reflection. We use the model of [8] where the ocean's albedo depends only on wind speed $W_{S}$ (which is given by the NASA meteorological database). The model divides the albedo into two components: one that is caused by direct light $A_{\text {dir }}$ and one by diffuse light $A_{\text {dif }}$. Defining $\mu_{0}=\sin h$, we use the reference wind speed in meters per second, $W_{0}(4)$.

$$
W_{0}=180 \mu_{0}^{3}\left(1-\mu_{0}^{2}\right)
$$

If $W_{s} \geq W_{0}$ :

$$
\begin{aligned}
A_{\text {dir }}\left(\mu_{0}, W_{s}\right)=A & +B\left(1-\mu_{0}\right)^{2}+C\left(1-\mu_{0}\right)^{3} \\
& -D\left(1-\mu_{0}\right)^{6} \\
& +\left[\frac{4}{E+W_{s}-W_{0}}\right. \\
& \left.+\frac{F\left(1-\mu_{0}\right)}{1+W_{s}-W_{0}}\right]\left(1-\mu_{0}\right)^{6}
\end{aligned}
$$

With: $A=0.0210 S I, B=0.0421 S I, C=0.128 S I$, $D=0.0400 S I, E=5.68 S I$ and $F=0.0740 S I$ 


$$
\text { If } W_{s} \leq W_{0} \text { : }
$$

$$
\begin{aligned}
& A_{d i r}\left(\mu_{0}, W_{s}\right) \\
& =\left[1+\frac{G \mu_{0}{ }^{2}\left(1-\mu_{0}{ }^{2)} W_{s}\left(W_{s}-1.1 W_{0}\right)^{2}\right.}{W_{0}{ }^{3}}\right][A \\
& +B\left(1-\mu_{0}\right)^{2}+C\left(1-\mu_{0}\right)^{3}-D\left(1-\mu_{0}\right)^{6} \\
& \left.+\left[\frac{4}{E}+F\left(1-\mu_{0}\right)\right]\left(1-\mu_{0}\right)^{6}\right]
\end{aligned}
$$

With: $G=5.40$ SI. In either case, the diffuse component is given in (7).

$$
A_{d i f}\left(\mu_{0}, W_{s}\right)=H\left[1+I e^{-\left(\frac{W_{s}}{7}\right)^{2}}+J e^{-\left(\frac{W_{s}}{40}\right)^{2}}\right]
$$

With: $H=0.022 S I, I=0.0550 S I$ and $J=1.45 S I$.

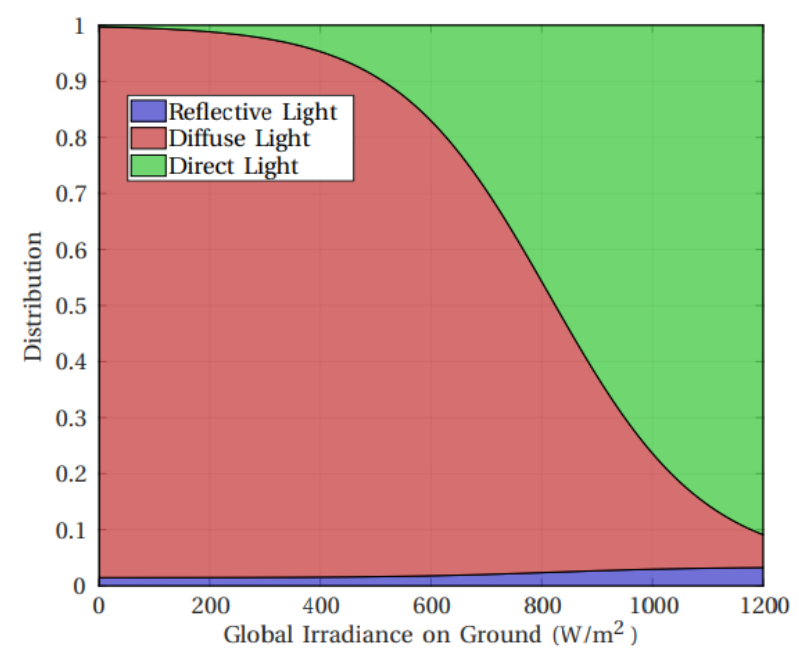

Fig. 3. The distribution of the three components of the light for a TOA irradiance of $1200 \mathrm{~W} / \mathrm{m}^{2}$

We then have (8).

$$
A=d_{t} A_{d i f}+\left(1-d_{t}\right) A_{d i r}
$$

Finally we have (9):

$$
A_{\text {total }}=A(1-f)+0.3 f
$$

With: $f=3.8410^{-6} W_{s}^{3.41}$.

The reflective component can then be computed using (10).

$$
I_{\text {reflect }}=A_{\text {total }} \sin h\left(\sin \frac{i}{2}\right)^{2} I_{0}
$$

\section{The resulting irradiance}

Finally, the irradiance of a single point on a solar cell is given in (11).

$$
\mathrm{I}_{\text {point }}=\mathrm{I}_{\text {direct }}+\mathrm{I}_{\text {diffuse }}+\mathrm{I}_{\text {reflect }}
$$

A distribution of those three components can be seen on the Fig. 3. On that figure, the top of atmosphere, TOA, irradiance is $1200 \mathrm{~W} / \mathrm{m}^{2}$ (the annual average is $1361 \mathrm{~W} / \mathrm{m}^{2}$ ) and the ground global irradiance varies from 0 to 1200 $\mathrm{W} / \mathrm{m}^{2}$. We can see that the diffuse light is predominant when the ground irradiance is below $800 \mathrm{~W} / \mathrm{m}^{2}$. We also see that the reflective component is always very low so that it might be negligible if needed.

To validate the model we compared its output with the irradiance measured between 2015 and 2016 at LAASCNRS, Toulouse, France, for a $45^{\circ}$ tilted panel toward South. The model uses weather database from NASA database. This database provides the top of atmosphere irradiation, the ground irradiation, the wind speed and the wind direction from the year 1997 to 2017 . The irradiation $E$ is the energy received during a day in $\mathrm{kWh}$. To get the irradiance $I$ we first consider the daily evolution of it as a sine arch that has an amplitude of $I$ and a period equal to two times the time between the sunrise and the sunset. The irradiance $I$ is given in (12).

$$
I=E \frac{\pi}{2} \frac{1}{t_{\text {sunset }}-t_{\text {sunrise }}}
$$

The comparison is shown in Fig. 4. The pink area covers data of the twenty years and in red the average value for each day. The green line exhibits data from the LAAS Laboratory. We can see that the model fit globally well except in winter where the diffuse component is greater and underestimated modeled. Moreover, we test the model on a daily basis for three days, from the 26th to the 28th of June 2018. The results (Fig. 5) show the effect of a very cloudy day on the irradiance but what matters for us is not the instantaneous power but the daily energy received (the irradiation). The relative error of the model is, for those three days, $7.5 \%, 5.5 \%$ and $10.6 \%$. The model is globally pessimist.

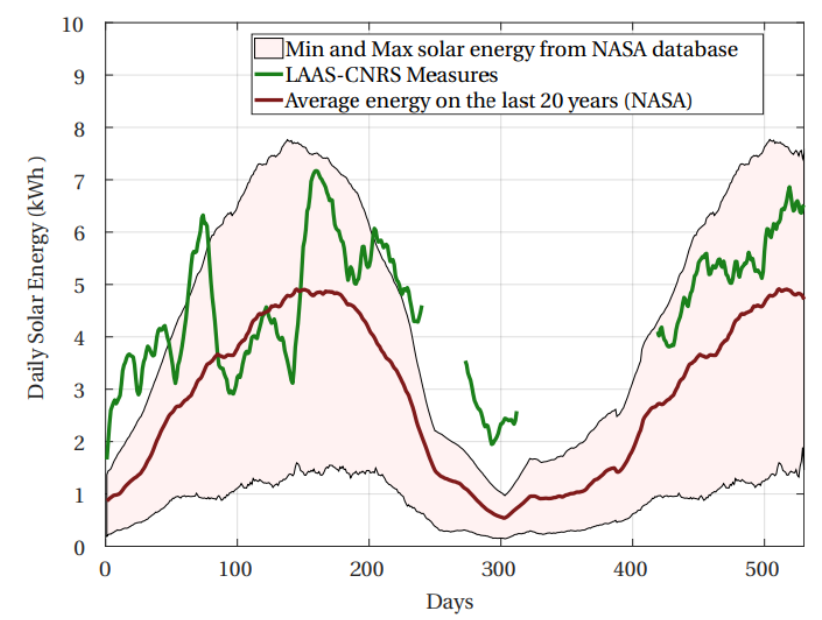

Fig. 4. Comparison of measured irradiation and the model for a $45^{\circ}$ tilted solar panel 


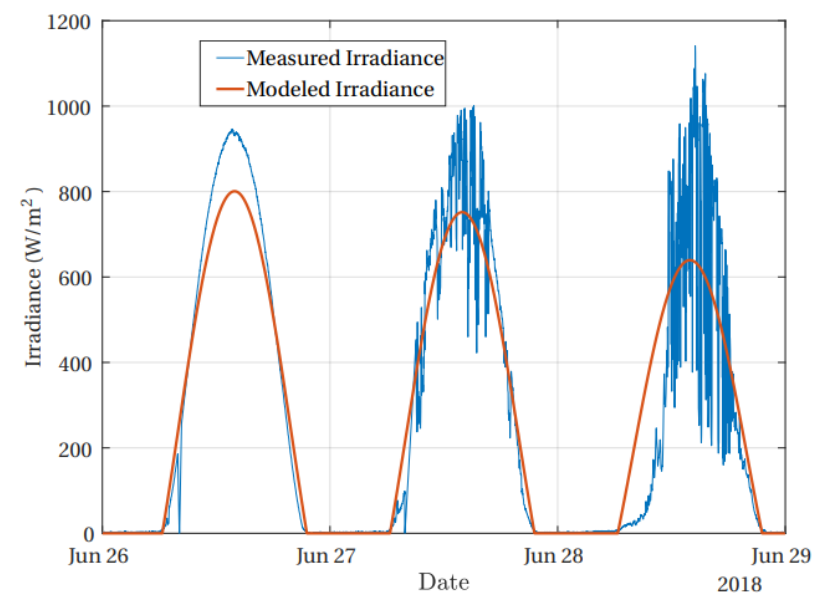

Fig. 5. Comparison of measured irradiance and the model for three days

\section{Computing the shadow of the sail}

The solar panel mounted is an 8 by 4 SunPower cells connected in series with no by-pass diode (Fig. 1), its radius of curvature is about $970 \mathrm{~mm}$ (the cells themselves are considered flat). The onboard computer constantly adapts the sail angle to the measured wind direction in order to maximize the speed [9]. Knowing the position of the boat and the time, the position of the sun is computed. The shadow of the sail on the cells can therefore be computed. The first solution was to compute for a certain amount of points of a cell if they are shaded or not. That was too long in calculation time. So the final solution was to pre-process the percentage of shade per cells for different relative positions of the sun and different sail positions using the method detailed below to build a lookup table. To determine this percentage we create a mesh of a cell, 15 by 15 grid (this discretization is a good compromise between precision and computation time), and for each point $\mathscr{M}$ we determine if the point is lighted by the sun using the following geometry protocol for a simple sail composed of an unique (for simplification) triangle $(A B C)$ :

- We define the unit radius sphere $\mathscr{S}$, centered on the point $\mathscr{M}$

- We project the triangle $(A B C)$ onto the sphere $\mathscr{S}$. We call the projected spherical triangle $\left(A^{\prime} B^{\prime} C^{\prime}\right)$, Fig. 6.

- The position of the Sun in the sky can be represented by a line that runs thought $\mathscr{M}$ and the Sun. We call the intersection of that line and the sphere $\mathscr{S}$ the point $S$, Fig. 6.

- $\quad$ The point $\mathscr{M}$ is shaded if and only if $S$ is inside the spherical triangle $\left(A^{\prime} B^{\prime} C^{\prime}\right)$.

A way to determine if the point is inside the spherical triangle is to check the relation (13) (areas are not Euclidean, they are spherical).

$$
\begin{gathered}
\text { Area }_{A^{\prime} B^{\prime} C^{\prime}}=\text { Area }_{S B^{\prime} C^{\prime}}+\text { Area }_{A^{\prime} S C^{\prime}} \\
+ \text { Area }_{A^{\prime} B^{\prime} S}
\end{gathered}
$$

To calculate the area of a spherical triangle $(A B C)$ we need the angles $a, b, c, \alpha, \beta$ and $\gamma$, Fig. 7. The first three angles are given by (14).

$$
\begin{aligned}
& a=\operatorname{atan} 2(|\overrightarrow{M B} \times \overrightarrow{M C}|, \overrightarrow{M B} \cdot \overrightarrow{M C}) \\
& b=\operatorname{atan} 2(|\overrightarrow{M C} \times \overrightarrow{M A}|, \overrightarrow{M C} \cdot \overrightarrow{M A}) \\
& c=\operatorname{atan} 2(|\overrightarrow{M A} \times \overrightarrow{M B}|, \overrightarrow{M A} \cdot \overrightarrow{M B})
\end{aligned}
$$

And the last three angles by (15).

$$
\begin{aligned}
& \alpha=\cos ^{-1}\left(\frac{\cos a-\cos b \cos c}{\sin b \sin c}\right) \\
& \beta=\cos ^{-1}\left(\frac{\cos a b-\cos c \cos a}{\sin c \sin a}\right) \\
& \gamma=\cos ^{-1}\left(\frac{\cos c-\cos a \cos b}{\sin a \sin b}\right)
\end{aligned}
$$

The final area of the triangle is then given by (16).

$$
\operatorname{Area}_{A B C}=\alpha+\beta+\gamma-\pi
$$

Then we calculate the ratio of shaded point to the total of points. To determine the solar irradiance by taking into account the sail we use (17), with $\sigma$ the percentage of not shaded cell.

$$
I_{\text {cell }}=I_{\text {diffuse }}+I_{\text {reflect }}+\sigma I_{\text {direct }}
$$

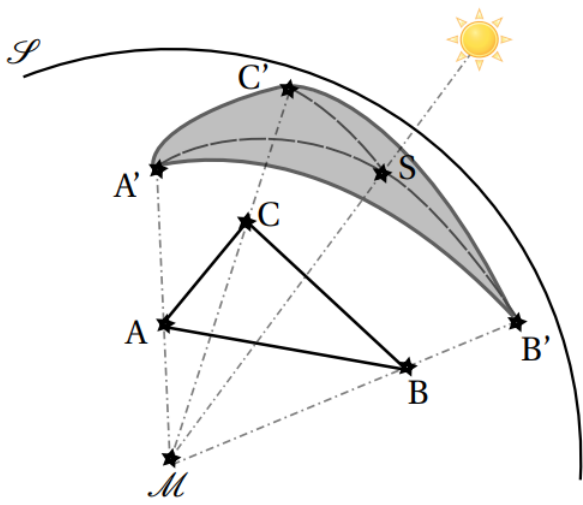

Fig. 6. Projection of a triangle on the sphere $S$

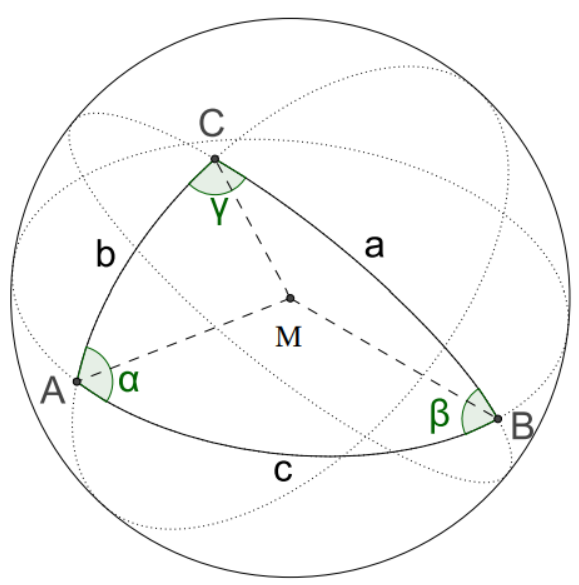

Fig. 7. Definition of the angles of a spherical triangle 


\section{Solar energy harvested by the solar panel}

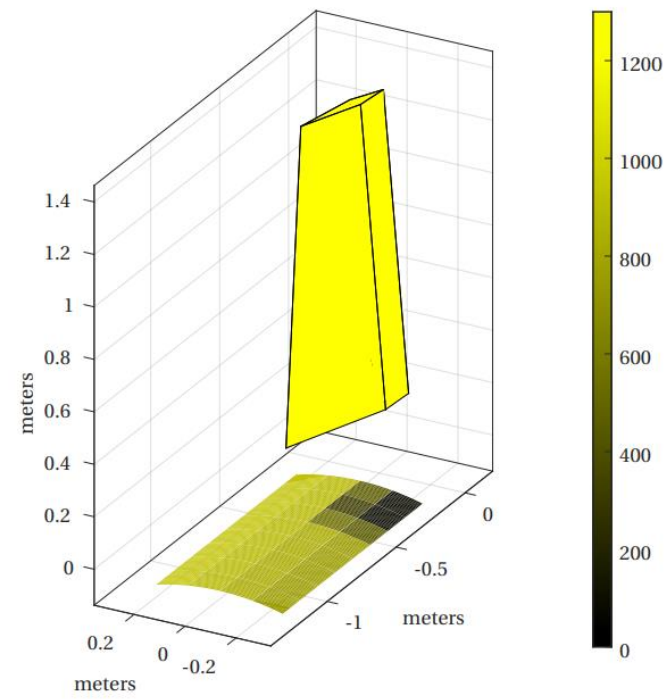

Fig. 8. Representation of the irradiance on each cells

We have now all the tools required to calculate the irradiance on each cell of the panel, the Fig. 8 shows a possible output (only the sail and the panel are represented). In this case the sail is made of a bunch of quadrilateral, each of them can be broken down into elementary triangles. We apply the previous steps to those triangles. To get the electrical power of each cell we only have to multiply it by the efficiency $\eta$ of the panel (about 13\%) and by its surface $S$ (32 square cells of $12.5 \mathrm{~cm}, 0.5 \mathrm{~m}^{2}$ ). The electrical power available at the panel connectors will depends on the connection of the cells, in the most basic configuration they are connected in series with no bypass diode then the power of the panel will be the power of the least powerful cell multiplied by the number of cells $N(18)$.

$$
P_{\text {panel }}=\eta N S \min _{n \in[1 . . N]}\left(I_{\text {cell } n}\right)
$$

To get the energy harvested during the $i$-th step time $\Delta t(i)$ of the simulation we then have to multiply the step time by the power of the panel (19). Finally, to know the daily energy of the day $j$ we sum each energy of the day (20). Of course, during the simulation one might takes care that the battery does not get more energy than it can handle.

$$
\begin{aligned}
& E_{\text {panel }}(i)=P_{\text {panel }}(i) \cdot \Delta \mathrm{t}(\mathrm{i}) \\
& E_{\text {panel }}(j)=\sum_{i=1}^{24} E_{\text {panel }}(i)
\end{aligned}
$$

\section{Boat behavior model}

To simulate the journey of the boat and the position of the sail we had to develop a model for its behavior. The model is based on data collected on the previous version of the boat. The speed of the boat (in meters per second), its list angle (in degrees, it is the angle to which the ship tilts either to port or starboard) and the position of the sail (in degrees) are then calculated with the wind speed and direction, and the heading of the boat relative to the wind (also called point of sail). Coefficients $\alpha, \beta, \gamma, \delta$ and $\kappa$ are computed with an optimization algorithm coupled with sailing data. We will not determine the model of the rolling of the boat as we suppose it to be periodic, and the final simulation will not be real-time. Instead, we will consider the rolling as a perfect sine wave of a random amplitude (from $0^{\circ}$ to $20^{\circ}$ ) and compute the irradiance on the panel for a certain amount of points along the rolling.

$$
\begin{gathered}
\text { Boat }_{\text {speed }}=\delta\left(\begin{array}{c}
\left.1-e^{1-\frac{\text { Wind }_{\text {speed }}}{\alpha}}\right)(1 \\
+\cos (\text { heading }-\pi))^{\beta}
\end{array}\right. \\
\text { Boat } \left._{\text {list }}=\kappa \text { Wind }_{\text {speed }}{ }^{\gamma} \text { (heading }-\pi\right) \frac{180}{\pi}
\end{gathered}
$$

The Figures 9 and 10 show the results of this model for different wind speeds in knots. The sail is a symmetrical airfoil (a NACA-0012 [10]). To determine its angle we use the $\boldsymbol{C}_{\boldsymbol{L}}(\boldsymbol{\alpha}) \boldsymbol{v} \boldsymbol{C}_{\boldsymbol{D}}(\boldsymbol{\alpha})$ (resp. lift and drag coefficient versus angle of attack $\alpha$ ) the airfoil. For each wind direction, we determine the angle of attack $\alpha$ that maximizes the aerodynamic force along the longitudinal axis of the boat.

\section{Simulating the journey}

Those models of irradiance and behavior have been implemented into a simulator built with MATLAB. A hundred of boats are simulated and each of them is assigned to a certain year among 1997 to 2017. The boats will then get the weather of the year they are assigned to with a $5 \%$ random noise added. Each of the boats have a $500 \mathrm{Wh}$ battery initially full and an average power consumption of $6 \mathrm{~W}$, the solar panel efficiency is set to $13 \%$. We present the results of two simulations: the first one is based on our accurate model; the second one uses a basic model (neglecting the sail's shadow and list angle).

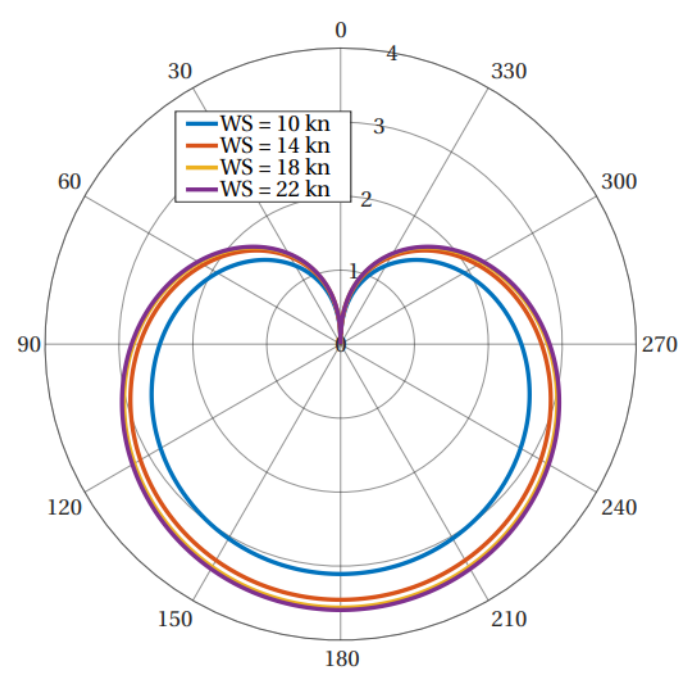

Fig. 9. Boat speed polar curve in meters per second determined by our model

Each simulation exhibits a hundred boats racing from France to French Caribbean. We then compare the average daily irradiation of the boats for each of the simulation on Fig. 11. On this graph, we can see that in both case the boats produce more than needed. However, the impact of the sail is not negligible. 


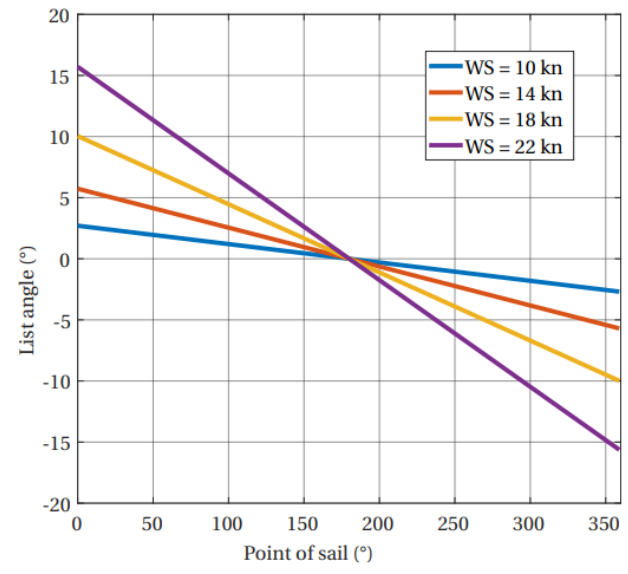

Fig. 10. List angle determined by our model

With the basic model, the boats produce an average of 280 $\mathrm{Wh} / \mathrm{d}$ versus an average of $210 \mathrm{Wh} / \mathrm{d}$ with the model we developed.

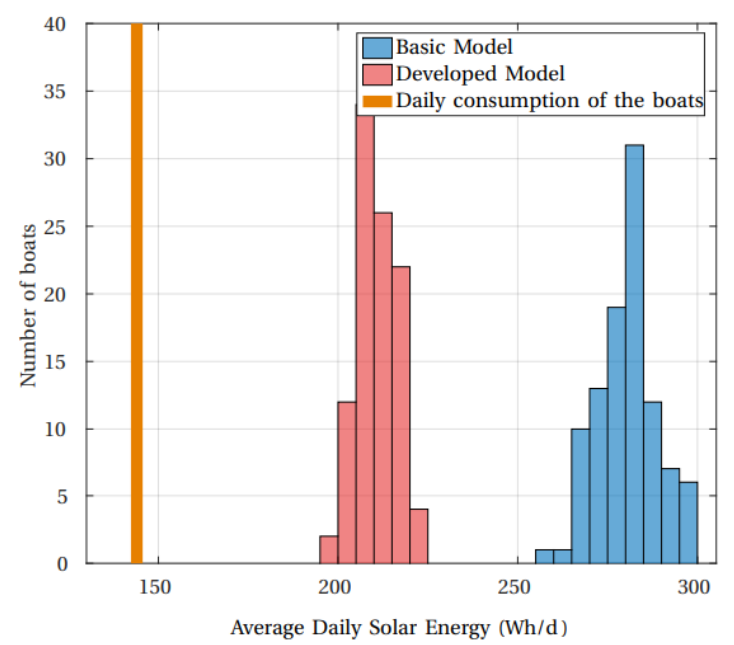

Fig. 11. Average daily solar energy of the boats for the developed model and for a basic model

\section{Conclusion}

The model developed in this study relies on two main components, the calculation of irradiance and the behavior of the boat. The accuracy of the irradiance model, $10 \%$ for a hazy sky, is good enough for the purpose of the study. In addition, the behavior model has been developed using actual data. So that we can suppose the whole model will fit correctly and will able us to determine the solar power available for a known situation (the boats direction and position, the winds speed and direction and the time). Unfortunately, we will not be able to verify it because the measure of power on the solar panel is not available while sailing. However, the model will be used in a decision support tool. It will be included in a simulator to help IBOAT3 developers to choose the path and the batteries size. Furthermore the methodology used in this study could be adapted by one for another project.

\section{References}

[1] Yves Briere. "Iboat: An autonomous robot for long-term offshore operation". In: Electrotechnical Conference, 2008. MELECON 2008. The 14th IEEE Mediterranean. IEEE. 2008, pp. 323-329.

[2] Microtransat Challenge. URL: www.microtransat.org

[3] R Maceiras et al. "Implementation of renewable energy systems on sailboats for auxiliary energy systems". In: ().

[4] Juraci Carlos de Castro Nóbrega and Andrej Rössling. "Development of solar powered boat for maximum energy efficiency". In: International Conference on Renewable Energies and Power Quality (ICREPQ'12), Santiago de Compostela (Spain), 28th to 30th March. 2012.

[5] Hiren Patel and Vivek Agarwal. "MATLAB-based modeling to study the effects of partial shading on PV array characteristics". In: (2008).

[6] John Boland, Barbara Ridley, and Bruce Brown. "Models of diffuse solar radiation”. In: Renewable Energy 33.4 (2008), pp. 575-584.

[7] Richard Perez et al. The development and verification of the Perez diffuse radiation model. Tech. rep. Sandia National Labs., Albuquerque, NM (USA); State Univ. of New York, Albany (USA). Atmospheric Sciences Research Center, 1988.

[8] J Li et al. "Ocean surface albedo and its impact on radiation balance in climate models". In: Journal of climate 19.24 (2006), pp. 6314-6333.

[9] Yves Briere. "Sailing robot performance: maximum speed tracking vs energy efficiency". In: Field Robotics. World Scientific, 2012, pp. 102-109.

[10] Airfoil Tools. URL: www.airfoiltools.com 